(C)2016 IEEE. Reprinted, with permission, from S.E. Hamada, I.A. Alshalabi, K.M. Elleithy, and I.A. Badara, "Automated Adaptive Mobile Learning System using the Semantic WEB.” In Proceedings of 2016 IEEE Long Island Systems, Applications and Technology Conference (LISAT), East Farmingdale, NY, 2016. DOI: 10.1109/LISAT.2016.7494150.

This material is posted here with permission of the IEEE. Such permission of the IEEE does not in any way imply IEEE endorsement of any of the University of Bridgeport's products or services. Internal or personal use of this material is permitted. However, permission to reprint/republish this material for advertising or promotional purposes or for creating new collective works for resale or redistribution must be obtained from the IEEE by writing to pubs-permissions@ieee.org. By choosing to view this document, you agree to all provisions of the copyright laws protecting it. 


\section{Automated Adaptive Mobile Learning System using the Semantic WEB}

\author{
Samir Hamada \\ Department of Computer \\ Science and Engineering \\ University of Bridgeport \\ Bridgeport- CT, USA \\ shamada@my.bridgeport.edu
}

\author{
Ibrahim Alkore Alshalabi \\ Department of Computer \\ Science and Engineering \\ University of Bridgeport \\ Bridgeport- CT, USA \\ ialkorea@my.bridgeport.edu
}

\author{
Khaled Elleithy \\ Department of Computer \\ Science and Engineering \\ University of Bridgeport \\ Bridgeport- CT, USA \\ elleithy@bridgeport.edu
}

\author{
Ioana A. Badara \\ School of Education University \\ of Bridgeport \\ Bridgeport- CT, USA \\ ibadara@bridgeport.edu
}

\begin{abstract}
ELearning (Electronic Learning) and mlearning (Mobile Learning) systems are online learning platforms. In our research we are modeling them as a weighted directed graph where each node represents a course unit. A directed graph represents an accurate picture of course descriptions for online courses through the computer-based implementation of various educational systems. The Learning Path Graph (LPG) represents and describes the structure of domain knowledge, including the learning goals, and all other available learning paths. In this paper, we propose an adaptive m-learning system architecture and a conceptual framework that uses the Semantic Web to obtain the students' data from other educational institutions. This process will enable the educational institutions to communicate and exchange students' data, and then use this information to adjust the students' profiles and modify their learning paths. The Semantic Web will create a more personalized dynamic course for individual students according to their ability, educational level, and experience.
\end{abstract}

Keywords: Adaptive Learning; dotNetRDF; Graph; M-learning; Ontology; RDF; Semantic Web; Shortest Path; Turtle; User Profile.

\section{INTRODUCTION}

Adaptive eLearning researchers explore and develop adaptive techniques that provide a better educational experience for students. Researchers offer accurate and personalized content to students in an effective way [1] that may allow for adjustments in course content based on the student's most recent performance. This technique allows the student to skip unnecessary and/or redundant learning activities by providing automated and personalized support for the student [2]. Students with different educational backgrounds and with different learning styles are the main challenge for the eLearning and m-learning systems. These systems provide personalized course units to meet the educational needs of different students.

Throughout the most recent decades, various research has examined the possibilities of changing the educational instruction model from the customary onesize-fits-all instructing model to a more adaptive and customized learning instruction model.
Most of the techniques calculate the optimal learning path depending on the characteristics in the student's profile to make the course more personalized. However, we have not seen any technique updating the profiles dynamically using the Semantic Web to exchange the information between educational institutions.

The student profile contains information about the student such as first name, last name, address, course units that have been completed, and grades of those course units.

This model can be applied for mobile leaning and eLearning in community colleges as well as in a typical graduate or under graduate course. Students can benefit from and personalize their college experience and completing their requirements. However this model does not apply to K-12 students as they are outside the scope of our research.

Adaptive Learning is an educational method that aids students in the learning process according to their needs. In addition, Adaptive Learning assists instructors in conveying course content to their students in a personalized manner based on the students' ability and background. Furthermore, from the developers view point Adaptive Learning is a technique using computers and other resources to assist in producing a better learning experience.

One of the most challenging tasks for Adaptive Mobile Learning is to create an Adaptive Course. Several researchers have used different techniques in order to make the course adaptive in terms of the course content and units. To the best of our knowledge, we have not seen any research that attempts to make the course adaptive in terms of previously completed materials by the students at another educational institution by using the Semantic Web to communicate directly with various educational institutions' systems to acquire the students' profiles.

According to our proposed system, at the time of course registration, the students complete their profile information. If there is a claim that the student has successfully completed a course unit at another educational institution, our system will run a query against the Semantic Web files which will be performed by using The SPARQL (SPARQL Protocol and RDF Query Language) [3], where (RDF) is the Resource Description which "is a general-purpose language for representing information in the Web" [4]. During our research, we ran the query against TURTLE files ("Terse 
RDF Triple Language, a concrete syntax for RDF" [5]) in another website to simulate the other educational institution, and we were able to obtain the students' profile and grades in that course unit. In the following paragraph we will explain our process in detail.

When students sign up and complete their profile information during the sign-up process, they include the completed course units from different educational institutions. The system then will query the Semantic Web files (TURTLE) of that institution to get the student's profile, verify the student's grade and determine if the student passed the course unit according to the passing grades imposed by the subject matter expert of each educational institution using our system. If there is no result to ensure that the student has passed one particular course unit in the past, the student will be presented with a quiz for this course unit. If the student successfully passes this quiz (it is a computerized quiz provided by our system), the course unit will be marked as completed. However, if the student does not pass the quiz, the student must go through the course unit's learning materials, and then re-take the quiz until the student successfully completes the course unit.

\section{RELATED WORK}

In 2011, Bhatia et al. [6], explains that the Semantic Web is an augmentation of the current Web, in which data is characterized to empower computers and individuals to work with better coordination. This coordination is taken into account in our study, as we plan to communicate with various educational institutions in order to verify the students' claims.

In 2013, Hadi et al. [7], stated that the internet changed the way we collect and deliver information. In their paper, they have expressed that the methodology of executing RDF queries against the Semantic Web information will require an exact match between the inquiry structure and the RDF content. They have addressed this problem by converting RDF content into a matrix of features and treated queries as a classification problem. They have effectively built up a working model framework that exhibited the appropriateness of their methodology. This approach was taken into consideration in our research because we use RDF queries against the Semantic Web data (but not applied yet).

In 2013, Soualah et al. [8], stated that new technical capacities exist in the area of learning because of the improvements to mobile phones and wireless technologies. They expressed that mobile learning (mlearning) is a natural extension of eLearning. It has the ability to make learning available on a wide scale because of the rapid advancements in the wireless technologies and the broad utilization of mobile devices. They also stated that learners have different backgrounds, objectives, and are located in different learning environments (heterogeneity of time, learning time, visual support, ambient noise, etc.). In summary, by having more information about the learners, we can adjust the learning strategies to satisfy every learner's needs.

The approach employed by Soualah et al., consists of two levels:
1. Semantic level aims to express semantic characteristics of learning contents and learner context.

2. Behavioral level provides users with only the most relevant information.

This approach makes use of learning practices already employed in eLearning systems, and adapts them to mLearning. It is this idea that is fundamental to our current work since the new technical capacities provides a greater amount of possible tools to enhance learning.

In 2014, Grivokostopoulou et al. [9], stated that eLearning frameworks are turning into crucial means of education delivery. Information delivery is one of the current work fundamentals. We would like to clarify the eLearning term which is defined as "eLearning is learning utilizing electronic technologies to access educational curriculum outside of a traditional classroom. In most cases it refers to a course, program or degree delivered completely online." [10].

In 2015 Walia et al. [11], stated that the Semantic Web approach to eLearning provides relevant and meaningful information to the learner. However, the human mind develops its own cognitive structure based on personal experiences and background. In this method of eLearning the Semantic Web is clear by adding the human conceptual representation and has a mechanism to use the learner profile and experience. Providing relevant and meaningful information to the learner are at the center of our research.

As previously mentioned, various related works have contributed to the foundation of our research. The following papers address security issues of the Semantic Web that are relevant to our research, since we have to secure sensitive data.

In their research, Kagal et al. [12], 2003 concluded that in order to secure the Semantic Web the following two fundamental parts are required. The first part calls for a semantic strategy that characterizes security necessities. While the second part consists of a distributed policy management approach. According to Kagal et al., 2003 in distributed policy management, each entity can determine its own particular strategy for security and privacy. It is essential for web entities to have the capacity to clearly express their security. In order to achieve this end goal, they utilize a policy language according to a semantic language to markup security information for web entities. Furthermore, Kagal and collaborators developed two based security frameworks that include one for distributed environments, and one for supply chain management.

In 2003, Thuraisingham's paper [13], provided an overview of the Semantic Web and discussed security issues. Furthermore, he stated that security must apply to all of the Semantic Web layers. Thuraisingham suggested that the security of the Semantic Web should start at the beginning of the project. In addition, he concluded that there are situations in which $100 \%$ security should be guaranteed, however he acknowledged that there are situations that do not require $100 \%$ security. 
At this stage we have not incorporated any security policy because it is not in the scope of our research. However, we intend to incorporate a security policy in a later stage of our research. In 2015, we demonstrated that the Learning Path Graph, which is a proficient representation of online courses in the computer based usage of an educational framework [14]. This adaptive learning system is displayed as weighted directed graphs, where each course unit is represented by a node in the graph as shown in Figure 1. The Learning Path Graph represents the structure of domain knowledge, learning goals, and all available learning paths.

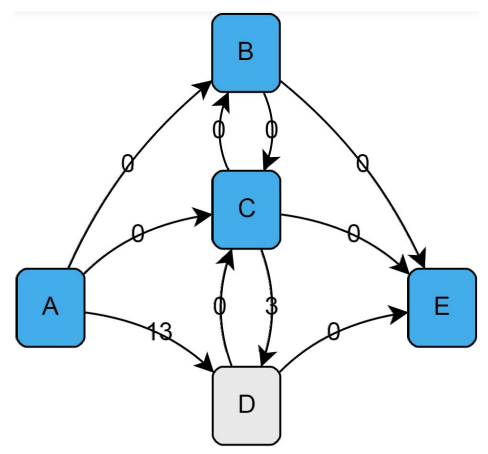

Figure 1. Learning Path Graph

In this study we implement an optimal adaptive learning path algorithm utilizing learner information from the learner's profile to enhance specific end goals. As well as to provide suitable content sequence in a dynamic structure for every learner [14]. This research intends to show how to optimize an Adaptive Mobile Learning System by using the Learning Path Graph (LPG). Furthermore, we intend to demonstrate how to customize the students' profiles by using the Semantic Web in order to provide credit to students for the course units completed in other accredited educational institutions. This research describes the conceptual framework of an Adaptive Mobile Learning System and how the students' profiles are used to adjust the learning path whereby making the learning path more dynamic. This means that when the student learns a course unit, there will be an adjustment to the learning path and a new optimal path will be generated. The interesting point in this research is the ability to use the Semantic Web to exchange the student's information among the educational institutions and to credit the students for the course unit that they have already completed. This feature may have the potential to boost the efficiency of adaptive learning systems and increase the chance for the student's success.

\section{ADAPTIVE M-LEARNING SYSTEM ARCHITECTURE}

An Adaptive m-learning system consists of several modules as shown in Figure 2. Typically, the System Interface contains an Admin Interface Module (AIM), an Instructor Interface Module (IIM) and Student Interface Module (SIM). This interface allows administrators, instructors and students to access our AML system.

A. System Interface.

1. Admin Interface Module (AIM)

2. Instructor Interface Module (IIM)

3. Student Interface Module (SIM)
B. Student Profile Module (SPM)
C. Learning Style Module (LSM)
D. Domain Concept Module (DCM)
E. Course Content Module (CCM)
F. Learning Path Generation Module (LPM)
G. Student Assessment Module (SAM)
H. Adaptive Engine Module (AEM)

The Adaptive Engine Module performs two tasks: the first task is to find all the personal learning paths using adaptive algorithms according to the student's profile. The second task is to retrieve the related teaching material according to the student's learning style.

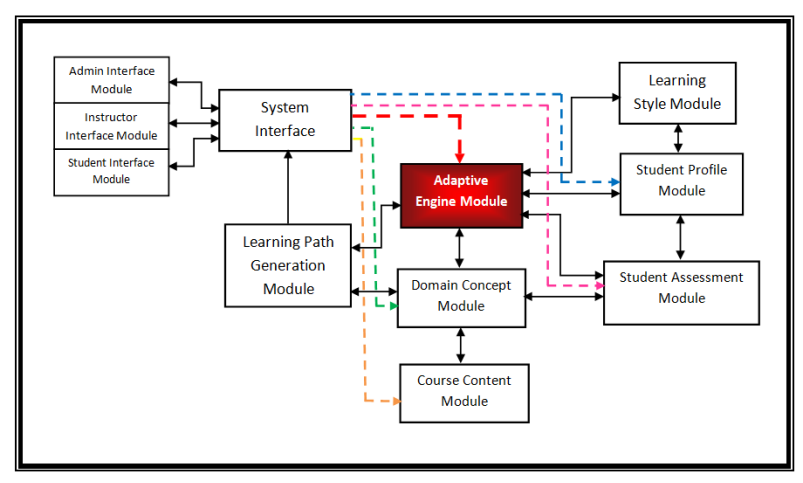

Figure 2. Adaptive m-learning system Architecture

\section{PROPOSED CONCEPTUAL FRAMEWORK}

Ontology as a term is derived from the Greek words onto, which means being, and the word logia, which means written or spoken discourse. Ontology means different things to different people. In philosophy, it represents the study of the existence and nature of being. In the Semantic Web, ontologies are formal definitions or representations of vocabularies or knowledge that allow the user to define resource classes, resource properties, and relationships between resource class members $[15,16]$.

Eisenstadt and Vincent [17] said that "An ontology is a partial specification of a conceptual vocabulary to be used for formulating knowledge-level theories about a domain of discourse." . As we can see in Figure 3 the three parts of the RDF Triple

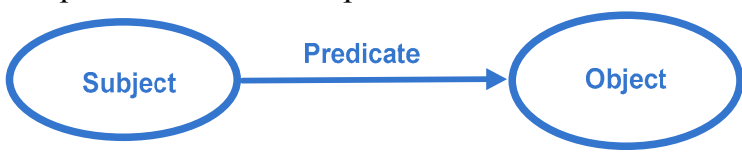

Figure 1- Resource Description Framework (RDF) Triple.

For example, as shown in Figure 4, the triple "(John) (Knows) (Jane)," (John) is the subject, (Knows) is the predicate, and (Jane) is the object.

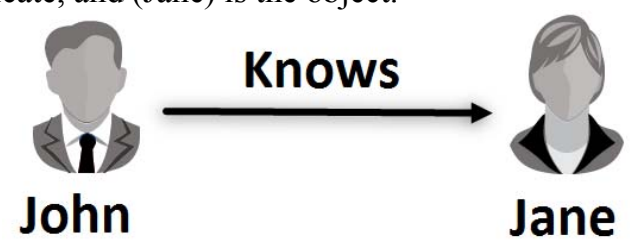

Figure 2-Parts of the Triple. 
Using TURTLE syntax, it can be written as shown in Figure 5.

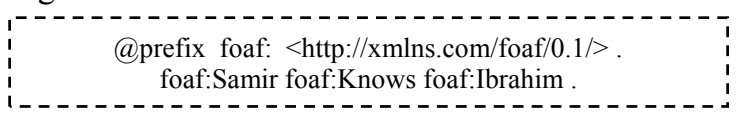

Figure 3 - Parts of the Triple in TURTLE format.

Advantages of using ontologies:

There are several advantages of ontologies including:

1. Publishing data using common vocabulary and grammar.

2. Preserving data semantic description is in ontologies

3. Data is ready for inference.

4. Better visibility.

5. Extensibility.

6. Flexibility.

7. Visibility

8. Inferenceability

9. Ability to add new properties at any time without breaking compatibility $[18,19]$.

Table 1 is a rough interpretation of terms used to describe relational databases and ontologies.

TABle 1- Relational DATABASE AND ONTOLOGY [19]

\begin{tabular}{|l|l|}
\hline \multicolumn{1}{|c|}{ Relational database } & \multicolumn{1}{c|}{ Ontology } \\
\hline row & subject \\
\hline column & predicate \\
\hline table data & literal nodes \\
\hline
\end{tabular}

The language that is used to query ontologies is SPARQL (SPARQL Protocol and RDF Query Language) which is a set of $\mathrm{W} 3 \mathrm{C}$ standards for querying and updating data conforming to the RDF (Resource Description Framework) model.[15]

In Figure 6 we show the student's properties.

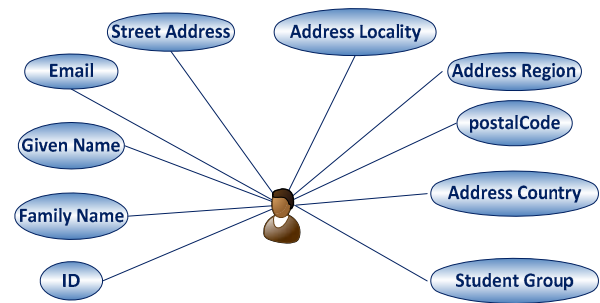

Figure 6 -Student's Properties

As we see in Figure each student has the following properties:

ID, Given Name, Family Name, Email, Street Address, Address Locality, Address Region, Postal Code, Address Country, and Student Group

In

Figure 7 we have the students' file in the TURTLE format. We have used some vocabulary from different schemas as follows:

@prefix d: <http://adaptivemobilelearning.com/ns/data\#>

@ prefix person: $<$ http://schema.org/Person $>$

@prefix address: <http://schema.org/>

@prefix place: $<$ http://schema.org/Place/>

(a)prefix aiiso: $<$ http://purl.org/vocab/aiiso/schema\#>

(a) prefix contains: $<$ http://schema.org/hasPart $>$

(a) prefix teach: <http://linkedscience.org/teach/ns\#>

(a)prefix completed: https://schema.org/Completed
Those are the available schemas that we were able to map our data files to, they might not be an exact match but that is not what our paper is studying, as our paper is a demonstration on how to obtain the student's results from another educational institution using the Semantic Web.

As shown if Figure 7 the students.ttl file.

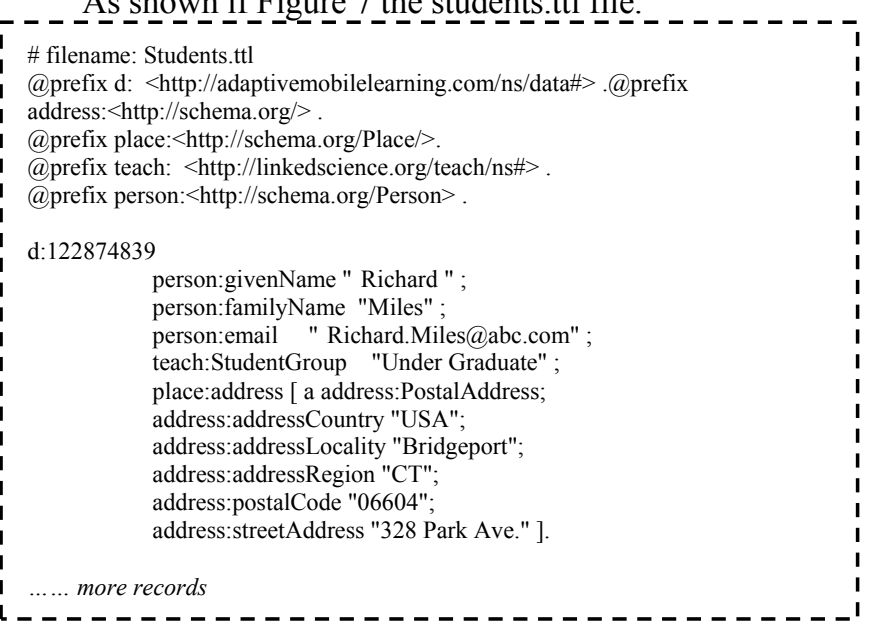

Figure 7 - Students' Data in TURTLE Format

We are going to use the SPARQL query to select students in $\mathrm{CT}$.

In Figure 8 we have a SPARQL query that is going to be run against thestudents.ttl TRUTLE file.

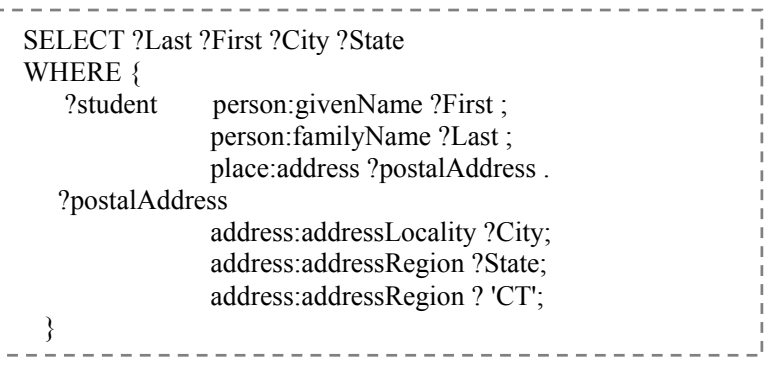

Figure 8 - SPARQL Query for students in CT

In Table 2 we can see the result of executing the query in Figure 8

Table 2 - Result of query from Figure

\begin{tabular}{|c|c|c|c|}
\hline Last & First & City & State \\
\hline Doe & John & Stratford & CT \\
\hline Miles & Richard & Bridgeport & CT \\
\hline
\end{tabular}

Let's add one more condition that the city is Stratford as shown if Figure 9.

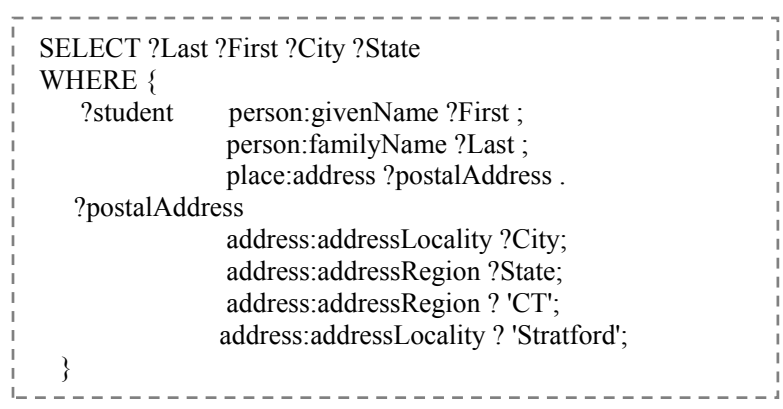

Figure 4 - Query for students in city $=$ Stratford and state $=\mathrm{CT}$ 
The only student in our student's table who lives in Stratford is John Doe as shown in table 3, and the result of running the query confirms that.

Table 3- Result of query from Figure 4

\begin{tabular}{|c|c|c|c|}
\hline Last & First & City & State \\
\hline Doe & John & Stratford & CT \\
\hline
\end{tabular}

\section{Implementing dotNetRDF $[14,15,20]$}

We used the dotNetRDF, were dotNetRDF Project aims to create an Open Source .Net Library using the latest versions of the .Net Framework for providing a powerful and easy to use API to work with RDF (Resource Description Framework), SPARQL and the Semantic Web. The primary goal is to provide an efficient method to work with reasonable amounts of RDF in .Net. Using dotNetRDF is extremely simple. Reading TURTLE files can be done as follows. The following snippet loads the Turtle files to an in-memory structured Graph. In Figure 10, Loading TURTLE files to memory.

using VDS.RDF;

using VDS.RDF.Parsing;

(..)

//Create a Symantic Web Graph

Graph g = new Graph();

UriLoader.Load(g, new

Uri("http://hamadafamily.com/sparq1/Faculty.ttl"));

(...)

Figure 10 - Loading the TURTLE files to memory

Here IN Figure 11, is the SPARQL Query that is going to be executed on the Graph $\mathrm{g}$.

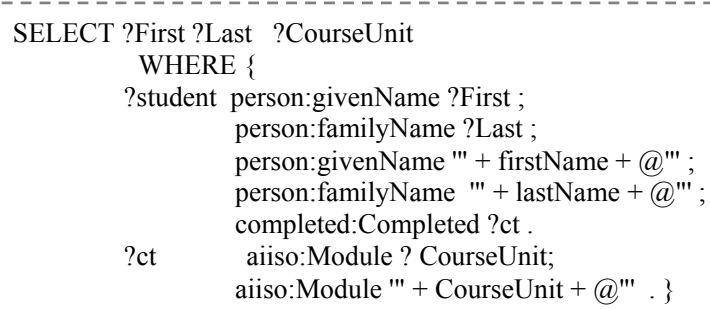

Figure 11 - A SPARQL query on the files that are loaded in Figure

The SPARQL queries can be executed with ExecuteQuery method as shown in Figure 12

//Query the data with SPARQL

Object results $=$ g.ExecuteQuery (query);

Figure 12 - Executing the query in Figure 11

The query is going to display Last Name, First Name and Course unit where first name equals Richard, Last Name equals Miles, and the course unit equals Introduction.
We were able to get the students information about the completed course units by supplying the parameters, firstName, lastName and course unit to the controller via the view and obtain data regarding if the course unit was passed by the student or not.

Then this information can be used to update the student's profile and then adjust the learning path to make it more adaptive according to the following system diagram Figure

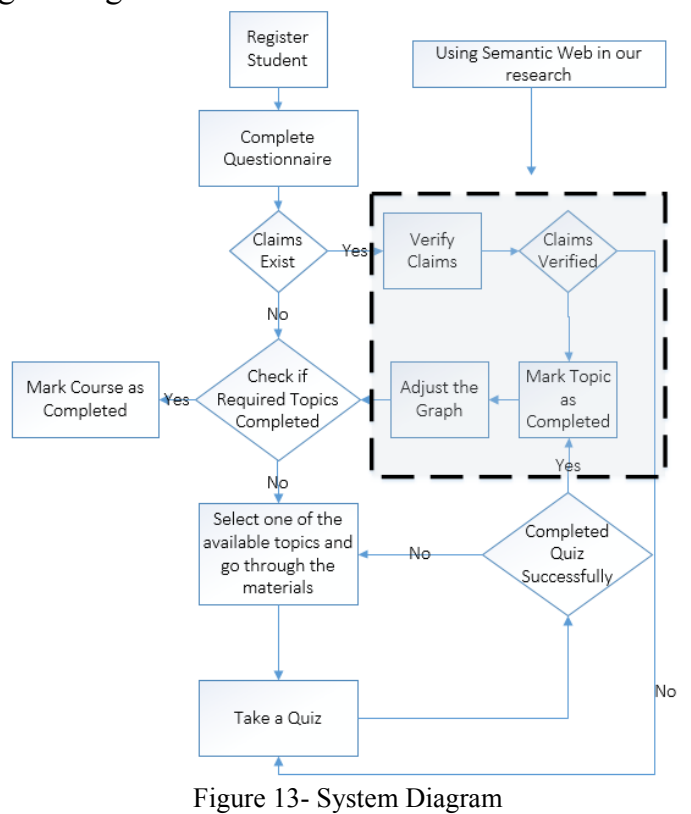

As we can see from Figure 13, when the student registers and complete the questionnaire if there are claims about successfully completing a course unit at another educational institution. The system will then query the TURTLE files located in that institution's website to verify the claim. Once the claim is verified the course unit will be marked as completed and then the system will check if the required course units to complete this course are successfully completed. The system then will mark the Course as completed otherwise the student has to complete the course unit quiz successfully in order to mark this course unit as completed, in case the student does not pass the quiz, the student will be able to select one of the available course units and go through its materials and then re-take the quiz. Upon passing it successfully, the course unit will be marked as completed and then the system will check if the required course units to complete this course were successfully completed then the system will mark the Couse as completed.

We have a student with the following attributes:

1. ID $=122874839$

2. Given Name $=$ "Richard"

3. Family Name = "Miles"

4. Email = "richard.miles@abc.com"

5. Student Group = "Under Graduate" 
Postal Address:

1. Street Address "328 Park Ave.

2. Address Locality = "Bridgeport"

3. Address Region $=$ "CT"

4. $\quad$ Postal Code $=$ "06604"

5. Country = "USA"

Richard Miles has completed the following course units:

1. Introduction

2. Arrays

The Introduction and Arrays are Parts Of course 390 which has the following attributes:

1. Study Program = "CPSC"

2. Course Title $=$ "Programming Pact"

3. Building = "Main Campus"

4. ects "Credits" = "6"

5. Student Group = "Undergraduate"

We can also query that the grade is in a specific acceptable range as described in The Semantic Web: Real-World Applications from Industry book to using RUD (University Resource Descriptor), SUD (Student University Descriptor), RQL (RDF Query Language), RDQL (RDF Data Query Language), SWRL (Semantic Web Rule Language) or Buchingae and SPARQL as shown in Figure 14, we can query the students with GPA $>3.5$ [21].

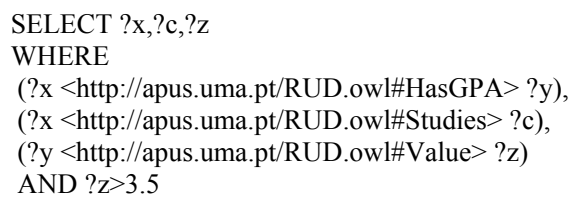

\section{CONCLUSION AND FUTURE DIRECTIONS}

This experiment has shown that we can use the Semantic Web with Adaptive Mobile Learning to enhance the courses making them more dynamic. The Semantic Web obtains the information about completed course units which are applied to the Learning Path Graph, and a new optimal path is generated. Furthermore, if the student completes the target module, the student does not have to complete the rest of the modules. We can also prevent teaching the same course unit for the student more than one time. Thus, the proposed approach can significantly improve the cost effectiveness for the students, and they can manage their time more efficiently.

Since there is a pre-test component to each course, we make sure that the students have the required knowledge needed in order to complete the course units.

The presented approach is expected to improve the performance of adaptive mobile learning and provides a learning experience to students that is more personalized and dynamic.

\section{REFERENCES}

[1] P. Sahai and M. Darbari, "Adaptive e-learning using Granulerised Agent Framework," International Journal of Scientific \& Engineering Research, vol. 5, 2014.

[2] J. Thompson. (2014, Adaptive Learning: Types of Adaptive Learning. Available:

http://teacherhelpguide.wikispaces.com/file/view/Adaptive +Learning+-Types+of+Adaptive+Learning.pdf

[3] W3.org. (25 Oct. 2015). SPARQL 1.1 Protocol. Available: http://www.w3.org/TR/sparql11-protocol/

(2016). RDF Current Status Available: https://www.w3.org/standards/techs/rdf\#w3c all

[5] (2016). Turtle - Terse RDF Triple Language. Available: https://www.w3.org/TeamSubmission/turtle/

[6] C. S. Bhatia and S. Jain, "Semantic Web Mining: Using Ontology Learning and Grammatical Rule Inference Technique," in 2011 International Conference on Process Automation, Control and Computing (PACC), 2011, pp. 16.

[7] A. S. Hadi, P. Fergus, C. Dobbins, and A. M. Al-Bakry, "A Machine Learning Algorithm for Searching Vectorised RDF Data," in 2013 27th International Conference on Advanced Information Networking and Applications Workshops (WAINA), 2013, pp. 613-618.

[8] F. Soualah-Alila, F. Mendes, and C. Nicolle, "A ContextBased Adaptation In Mobile Learning," IEEE Computer Society Technical Committee on Learning Technology (TCLT), vol. 15, p. 5 pages, 2013.

[9] F. Grivokostopoulou, I. Perikos, and I. Hatzilygeroudis, "Utilizing semantic web technologies and data mining techniques to analyze students learning and predict final performance," in 2014 International Conference on Teaching, Assessment and Learning (TALE), 2014, pp. 488494.

[10] (20 Mar. 2016). Erasmus+ - EACEA - European Commission. Available: https://eacea.ec.europa.eu/erasmus-plus en

[11] A. Walia, N. Singhal, and A. K. Sharma, "A Novel Elearning Approach to Add More Cognition to Semantic Web," in 2015 IEEE International Conference on Computational Intelligence \& Communication Technology (CICT), 2015, pp. 13-17.

[12] L. Kagal, T. Finin, and A. Joshi, "A policy based approach to security for the semantic web," in International Semantic Web Conference, 2003, pp. 402-418.

[13] B. Thuraisingham, "Security issues for the semantic Web," in 27th Annual International Computer Software and Applications Conference (COMPSAC), 2003, pp. 633-638.

[14] I. A. Alshalabi, S. Hamada, and K. Elleithy, "Automated adaptive learning using smart shortest path algorithm for course units," in 2015 IEEE Long Island Systems, Applications and Technology Conference (LISAT 2015), 2015, pp. 1-5.

[15] B. DuCharme, Learning Sparql: O'Reilly Media, Inc., 2013.

[16] J. H. Sofia, B. D. Sofia, A. D. José, and J. H. Leontios, Fuzzy Logic-Based Modeling in Collaborative and Blended Learning. Hershey, PA, USA: IGI Global, 2015.

[17] M. Eisenstadt and T. Vincent, The knowledge web: Learning and collaborating on the net: Routledge, 2012.

[18] L. Ding, P. Kolari, Z. Ding, and S. Avancha, "Using ontologies in the semantic web: A survey," in Ontologies, ed: Springer, 2007, pp. 79-113.

[19] J. Gajek. (28 Oct. 2015). Ontology, Notation 3, and SPARQL. Available: http://www.codeproject.com/Articles/156888/OntologyNotation-and-SPARQL

[20] (14 Apr. 2016). dotNetRDF - Semantic Web, RDF and SPARQL Library for C\#/.Net. Available: http://dotnetrdf.org/

[21] J. Cardoso, "Developing course management systems using the Semantic Web," in The Semantic Web. vol. Chapter 10, ed: Springer, 2008, pp. 169-188. 
Samir Hamada received the B.Sc. in Accounting from Ain Shams University, Cairo, Egypt in 1989, and the Master Degree of Computer Science from University of Bridgeport, Connecticut-USA in 2001; in 2008 he joined University of Bridgeport as Ph.D. Student in computer science and engineering at the University of Bridgeport, Connecticut-USA. Worked as an IT Manager for Atlantica Insurance Firm formally known as Action Auto Insurance From 2003 to Present, Samir thought the Data Computer Communication in the Spring and Fall of 2010, He also thought the course of Pre Calculus in the spring of 2011 and on Spring $2014 \mathrm{C}++$ and Data Structure, and currently teaching Mobile Communication, $\mathrm{C}++$ and Data Structure. Worked in many Web development projects for Boehringer Ingelheim USA Corp, Publics Modem, Modem Media, Noble Americas, Dakota, Osprey, Byte Inter Active, Visual Concepts, Family Time, He was the web master for Connecticut Golf Club from 2005 till 2010.He was the only student to receive the First Info Tech scholarship at the University of Bridgeport at the year of 2000 . He also received the General Diploma for Management Sciences in 1993 from the Sadat Academy, Cairo Egypt.

Ibrahim M Alkore Alshalabi received the B.Sc. in Computer Science from Al-Isra Private University, Amman, Jordan in 1997, and the MCA( Master of Computer Applications ) from Bangalore University India in 2007. In 2009 he joined University of Bridgeport as Ph.D. student in computer science and engineering at the University of Bridgeport, Connecticut-USA. From 1997 to 2004, he was Assistant Lecturer in Ma'an Community College - Al-Balqa Applied UniversityJordan. From 2007 to 2009 he joined Al-Hussein Bin Talal University-Jordan as assistant lecturer. Ibrahim M Alkore Alshalabi has research interest is in the general area of E-Learning, M-Learning, wireless communications and networks.He actively participated as a committee member of International Conference on Engineering Education, instructional technology, Assessment, and E-Learning (EIAE 10, EIAE 11).

Ibrahim $\mathrm{M}$ Alkore Alshalabi is a member of the International Joint Conferences on Computer, Information, and Systems Sciences, and Engineering (CISSE) and Ibrahim M Alkore Alshalabi is a member of IEEE.
Dr. Khaled Elleithy is the Associate Vice President for Graduate Studies and Research at the University of Bridgeport. He is a professor of Computer Science and Engineering. He has research interests in the areas of wireless sensor networks, mobile communications, network security, quantum computing, and formal approaches for design and verification. He has published more than three hundreds research papers in international journals and conferences in his areas of expertise.

Dr. Elleithy is the editor or co-editor for 12 books by Springer. $\mathrm{He}$ is a member of technical program committees of many international conferences as recognition of his research qualifications. He served as a guest editor for several International Journals. He was the chairperson for the International Conference on Industrial Electronics, Technology \& Automation, IETA 2001, 19-21 December 2001, Cairo - Egypt. Also, he is the General Chair of the 2005, 2006, 2007, 2008, 2009, 2010, 2011, 2012, 2013, and 2014 International Joint Conferences on Computer, Information, and Systems Sciences, and Engineering virtual conferences.

Dr. Joanna A. Badara holds a Ph.D. (Teacher Preparation/Science Education) from University of Tennessee-Knoxville and an M.Phil. (Microbiology \& Immunology) from University of Edinburgh, Scotland. Prior to completing her doctoral work, she has worked as a research scientist in the biomedical field for about ten years, having been affiliated with Weill Medical College of Cornell University and Mount Sinai School of Medicine, in New York City. Her passionate interest in the exploration of connections between scientists' epistemologies and the teaching of science led her to pursuing doctoral studies in Science Education. She has taught a multitude of core Biology courses for Biology/Pre-Medical undergraduates and mentored student research projects in this field. Dr. Badara is currently a faculty member at University of Bridgeport, where she teaches core research courses in the doctoral (Ed.D.) program, Science Education courses in the Science Teacher Preparation program, and History and Philosophy of Science courses at the undergraduate level. She has been the recipient of several grants for research, including a National Science Foundation grant for conducting research on the teaching of science in urban school districts. She has presented her work at national and international conferences in the field of STEM education. 